11

\title{
Релятивистский твистрон с КПД $56 \%$ на основе конструкции лампы обратной волны с модулирующим рефлектором
}

\author{
(C) Е.М. Тотьменинов, ${ }^{1}$ И.В. Пегель, ${ }^{1,2}$ В.П. Тараканов ${ }^{3,4}$ \\ ${ }^{1}$ Институт сильноточной электроники РАН, \\ 634055 Томск, Россия \\ ${ }^{2}$ Национальный исследовательский Томский политехнический университет, \\ 634050 Томск, Россия \\ ${ }^{3}$ Объединенный институт высоких температур РАН, \\ 125412 Москва, Россия \\ ${ }^{4}$ Национальный исследовательский ядерный университет „МИФИ“, \\ 115409 Москва, Россия \\ e-mail: totm@|fe.hcei.tsc.ru
}

(Поступило в Редакцию 7 июля 2016 г.)

Посредством численного моделирования продемонстрирован режим работы релятивистского черенковского СВЧ генератора типа твистрон на основе замедляющей системы лампы обратной волны и модулирующего рефлектора с немонотонным, сильно неоднородным по длине системы энергообменом и эффективностью преобразования по мощности от электронного пучка в электромагнитное излучение $56 \%$ при ,электронном“ КПД 66\%. Для ускоряющего напряжения $340 \mathrm{kV}$ и тока электронного пучка $3.3 \mathrm{kA}$ расчетная мощность генерации составила $630 \mathrm{MW}$ на частоте $9.7 \mathrm{GHz}$ при ведущем магнитном поле $2.2 \mathrm{~T}$.

DOI: $10.21883 /$ JTF.2017.06.44522.1978

Распространенным типом мощных СВЧ генераторов на основе сильноточных электронных пучков являются генераторы на основе релятивистской лампы обратной волны (РЛОВ). Внимание исследователей и разработчиков в последние два десятилетия привлекают РЛОВ, снабженные модулирующим рефлектором [1], обладающие кроме высокого КПД (свыше 40\%) и другими преимуществами: увеличенным размером и электропрочностью замедляющей системы (3С), возможностью в широких пределах управлять частотой генерации. Повышению КПД генераторов такого типа уделяется пристальное внимание. Ориентиром, стимулирующим эти исследования, являются высокие (до 60-70\%) величины КПД, полученные в разные годы в упрощенных моделях РЛОВ с ЗС, неоднородными по фазовой скорости или по связи волны с пучком [2,3]. Недавно в численном эксперименте с использованием полностью электромагнитного кода UNIPIC за счет сложного профилирования 3С прибора был рассчитан режим генерации РЛОВ с эффективностью 48\% [4].

В РЛОВ с модулирующим рефлектором [1] поле в рефлекторе вызывает модуляцию электронного пучка по энергии, и на вход ЗС поступает уже в большей или меньшей мере сгруппированный пучок. Было показано, что в приборе такого типа варьирование амплитуднофазовых соотношений между высокочастотным током в пучке, созданным модулятором, и полем синхронной волны на входе ЗС позволяет в широких пределах управлять частотой и эффективности генерации. В работе [1] подробно исследован случай, когда сгусток электронов на входе в ЗС попадает в тормозящую фазу синхронной $(-1)$ гармоники встречной волны, и ключевую роль в энергообмене играет взаимодействие пучка с полем именно этой гармоники. Для такого типа фазировки сгустка простые расчеты в одномерных моделях демонстрируют возможность достижения (при оптимальной величине объемного заряда пучка) высоких КПД - около 60\%.

Нельзя исключать, что высокоэффективая генерация возможна и при существенно иных амплитудно-фазовых соотношениях. Поиск в этом направлении может быть связан, в частности, с использованием полей наиболее интенсивных несинхронных волновых гармоник, существующих в 3С: нулевых гармоник попутной (и, возможно, встречной) волны и $(-1)$-й гармоники попутной волны. Расчет такого прибора и описан в настоящей работе.

Расчеты выполнены в осесимметричной версии полностью электромагнитного кода KARAT [5], ранее многократно использованного при разработке СВЧ генераторов различных типов [6-8], а также в одномерной PICмодели РЛОВ [9] с учетом объемного заряда пучка и названных выше волновых гармоник. Также использована программа для расчета волновых полей на основе метода матриц рассеяния [10].

Конфигурация СВЧ генератора изображена на рис. 1, a. Прибор имеет малую (сопоставимую с длиной волны излучения) протяженность участка взаимодействия пучка с волновым полем в пределах $3 \mathrm{C}$ $\left(L_{1}=36 \mathrm{~mm}\right)$, большую крутизну стенки волновода в месте высадки электронного пучка, правее которого имеется протяженный цилиндрический участок $L_{2}$. Расчетная эффективность преобразования мощности электронного пучка в электромагнитное излучение волны $\mathrm{TM}_{01}$ со- 

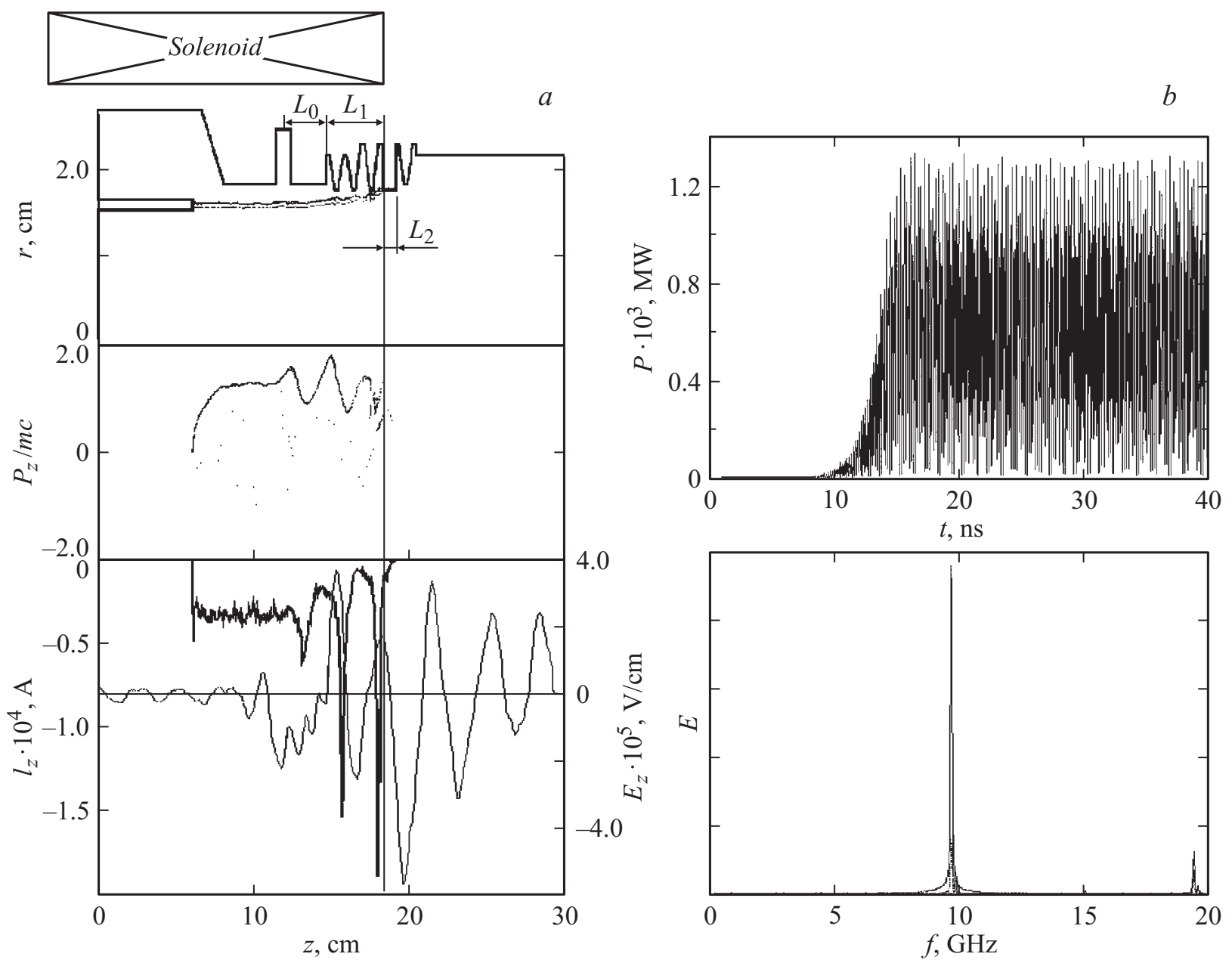

$b$

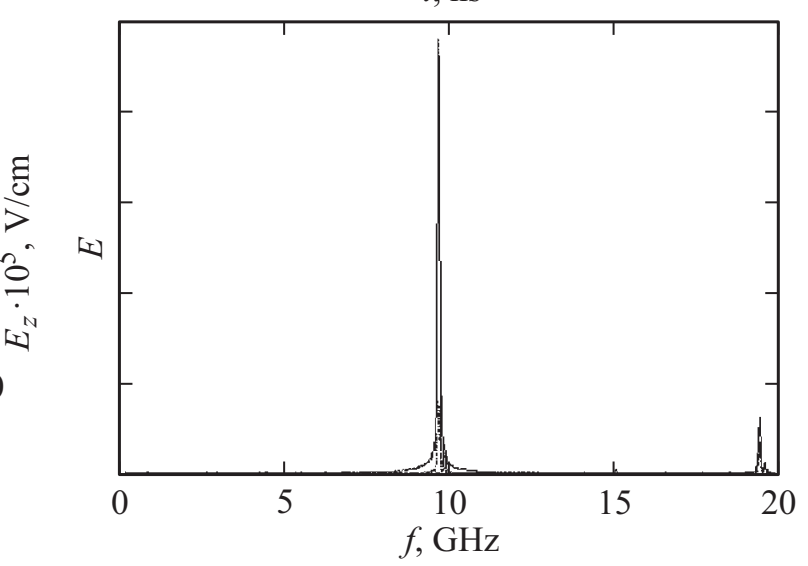

Рис. 1. $а$ - конфигурация прибора, фазовый портрет электронного пучка и зависимости тока пучка и $z$-компоненты электрического ВЧ поля от продольной координаты на оси системы в один из моментов времени (расчет PIC-кодом KARAT); $b$ - зависимость СВЧ мощности от времени и спектр генерации при ускоряющем напряжении $340 \mathrm{kV}$, токе пучка $3.3 \mathrm{kA}$, ведущем магнитном поле 2.2 Т. Мощность стационарной генерации $630 \mathrm{MW}$.

ставила $56 \%$ (рис. $1, b)$, а „электронный КПД“, рассчитанный по изменению кинетической энергии электронов относительно их энергии в канале перед рефлектором, $66 \%$ (рис. 2).

Фазировка сгустка в генераторе отличается от случая, проанализированного в [1]. Оценим его положение относительно синхронной $(-1)$-й гармоники встречной волны и (0)-й гармоники попутной волны на входе в 3 С. Ранее для случая, когда гофрировка начинается с максимального радиуса, было записано выражение для фазы модуляции, которая определяет положение центрального электрона сгустка относительно фазы продольной компоненты электрического поля $(-1)$-й гармоники на входе в 3С [1]:

$$
\arg (\alpha) \approx-\frac{\pi}{2}-\left(k \frac{L_{0}}{\beta_{\|}}+h L_{0}\right)+2 \pi m, \quad m=0,1, \ldots,
$$

где $h$ - продольное волновое число рабочей волны $\mathrm{TM}_{01}$ в волноводе между рефлектором и $3 \mathrm{C}, \beta_{\|}-$ продольная скорость электронов в единицах скорости света в вакууме. Здесь первое слагаемое соответствует разности фаз модулирующего поля и поля встречной волны в центральной плоскости рефлектора $(-\pi / 2$ соответствует середине ускоряющей фазы поля встречной волны). Поскольку отражение встречной волны от рефлектора происходит в режиме холостого хода [1], можно записать аналогичное выражение для основной гармоники попутной волны

$$
\arg (\beta) \approx \frac{\pi}{2}+\left(k \frac{L_{0}}{\beta_{\|}}-h L_{0}\right)+2 \pi n, \quad n=0,1, \ldots .
$$

Данный параметр определяет положение центрального электрона относительно фазы поля продольной компоненты электрического поля (0)-й гармоники попутной волны на входе в ЗС. Для параметров численного эксперимента (частота генерации $9.7 \mathrm{GHz}, L_{0}=27 \mathrm{~mm}$ ) имеем $\arg (\alpha) \approx 0$ и $\arg (\beta) \approx 1.4 \pi$. Это означает, что в месте входа в ЗС центральный электрон сгустка попадает на 


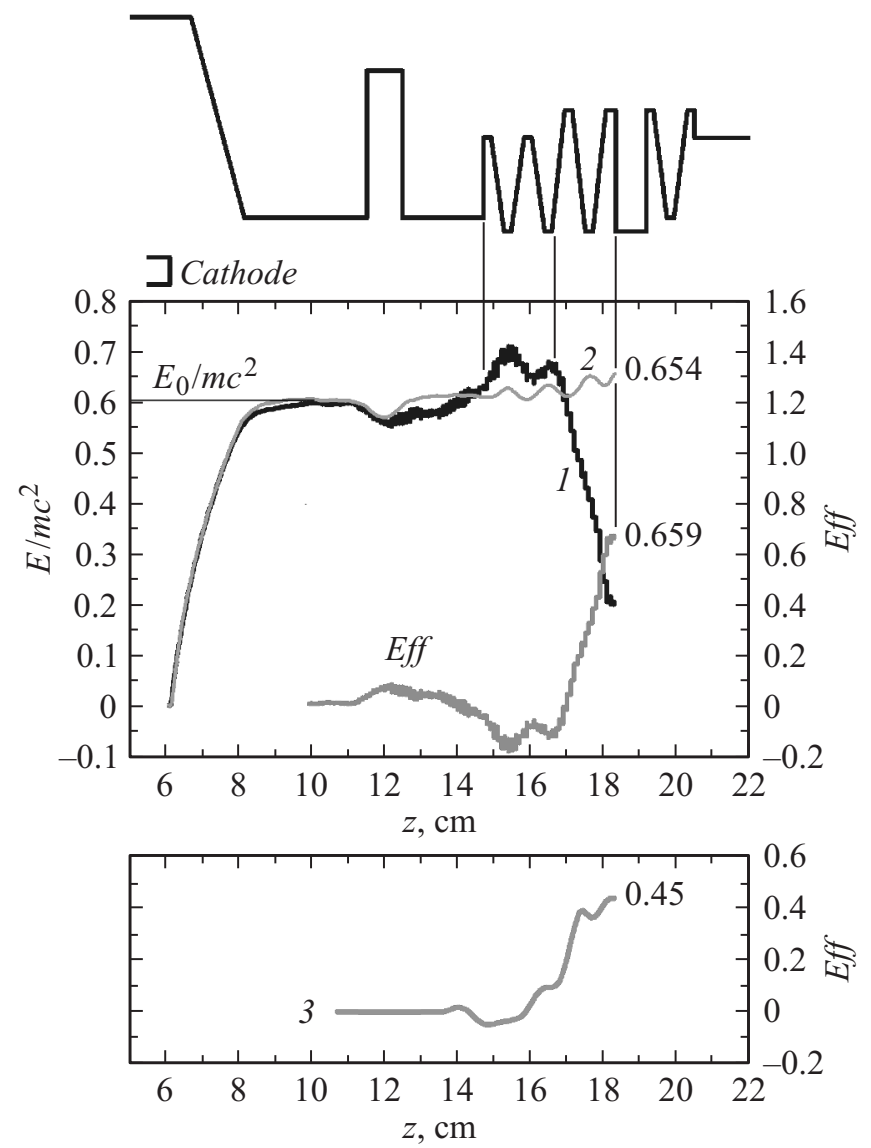

Рис. 2. Усредненные за период ВЧ колебаний: нормированная кинетическая энергия электронов $(1-$ в режиме стационарной СВЧ генерации, 2 - в отсутствие генерации) и „электронный КПД“, 3 - „электронный КПД“, полученный в одномерной модели без учета доускорения сгустка в конце ЗС. границу перехода от тормозящей к ускоряющей фазе поля синхронной $(-1)$-й пространственной гармоники рабочей волны и вблизи середины ускоряющей фазы поля (0-й) гармоники попутной волны. Кинематическое смещение центрального электрона сгустка относительно фазы поля основной гармоники попутной волны на участке $L_{1}=36 \mathrm{~mm}$ от входа в ЗС до места высадки пучка

$$
\Delta \phi_{0}=k L_{1}\left(\frac{1}{\beta_{\|}}-\frac{1}{\beta_{p h, 0}}\right) \approx \pi
$$

$\left(\beta_{p h, 0} \approx 1.14-\right.$ нормированная на скорость света фазовая скорость основной гармоники на участке 3 ). Тогда $\arg (\beta)+\Delta \phi_{0} \approx 2.4 \pi$. Следовательно, в конце пространства взаимодействия центральный электрон попадет в тормозящую фазу поля попутной волны (рис. 1,a). При этом центральный электрон все время находится в тормозящей фазе $(-1)$-й гармоники встречной волны, так как скорость электронов $\beta_{\|} \approx 0.77$ превышает ее фазовую скорость $\beta_{p h,-1} \approx 0.6$. Таким образом, вблизи места высадки сгустка тормозящее ВЧ поле представлено синфазной суммой двух основных гармоник.

При такой фазировке сгустка энергообмен немонотонен по длине взаимодействия (рис. 2). Сначала происходит доускорение сгустка полем нулевой гармоники попутной волны, в результате которого он смещается к середине тормозящей фазы $(-1)$-й гармоники встречной волны. С этого момента сгусток начинает отдавать энергию. Интенсивность энергоотбора достигает максимума вблизи места высадки пучка, поскольку связь пучка с продольным электрическим полем здесь максимальна вследствие крутого (нормального) наклона поверхности гофры.
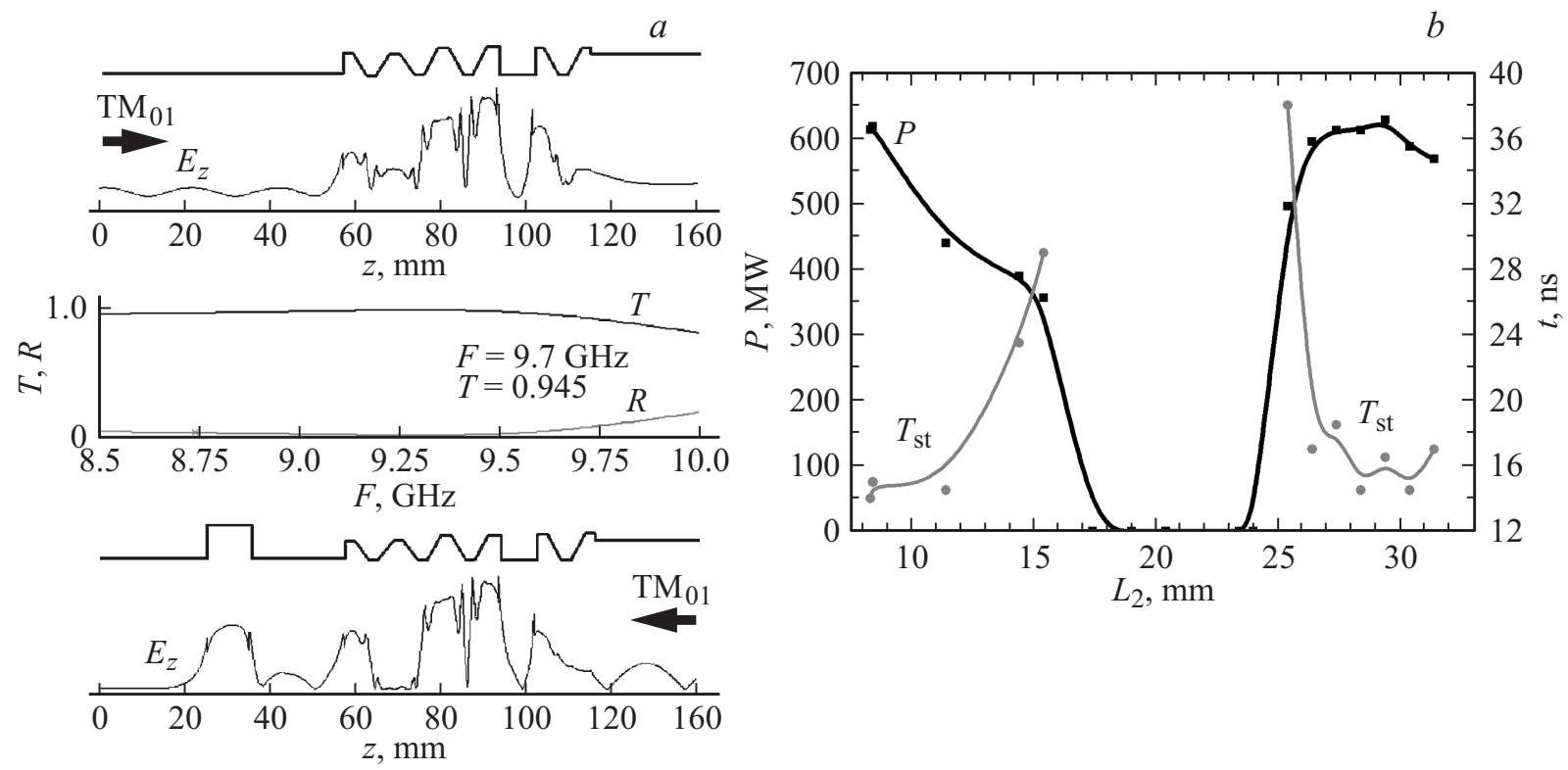

Рис. 3. $a-$ холодные распределения $z$-компоненты электрического поля на радиусе пучка (16 mm) и полосовая характеристика 3С генератора (коэффициент отражения $R$ и прохождения $T$ по амплитуде); $b$ - зависимость мощности и времени переходного процесса от параметра $L_{2}$. 
Сгусток электронов достигает наибольшей компактности непосредственно перед осаждением на поверхность 3С (рис. 1, $a$ ). В этом существенное отличие от описанного в [1] и в общем характерного для РЛОВ с протяженными 3С режима, когда сгустки, достигшие компактности и подверженные разрушению силами объемного заряда, существуют на большей части длины ЗС. По этой причине эффективность рассматриваемого прибора гораздо менее чувствительна к параметру объемного заряда. В частности, это проявляется в практическом отсутствии на фазовом портрете отраженных электронов, которые обычно в большом количестве возникают при разрушении сгустка объемным зарядом (процесс аналогичен образованию виртуального катода). Заметим, что отраженные электроны могут эффективно обмениваться энергией с полем $(-1)$-й гармоники попутной волны, что при их значительном количестве может влиять на КПД прибора.

Дополнительный положительный эффект, вызываемый объемным зарядом в рассматриваемой конфигурации, связан с тем, что поле объемного заряда доускоряет сгусток, теряющий скорость под действием интенсивного ВЧ поля перед высадкой на поверхность ЗС. Вследствие этого сгусток удерживается в тормозящей фазе ВЧ поля, a его потенциальная энергия переводится в энергию излучения.

Важную роль играет электродинамика структуры. Поскольку длина пространства взаимодействия невелика, для того чтобы обеспечить старт генератора, обеспечивается дозированное (около $10 \%$ по мощности) отражение СВЧ мощности от выхода ЗС (рис. 3, $a$ ). При изменении параметра $L_{2}$ происходит периодическое изменение мощности генерации и времени переходного процесса (рис. $3, b$ ). Периодичность максимумов СВЧ мощности по параметру $L_{2}$ составляет $21 \mathrm{~mm}$, что близко к половине волноводной длины волны $\mathrm{TM}_{01}$ на гладком участке $L_{2}$. Режимам с максимальной мощностью 620-630 MW соответствует минимальное время выхода на стационарный режим 15-18 ns. При сокращении величины $L_{2}$ менее $8 \mathrm{~mm}$ длительность переходного процесса резко возрастает.

Таким образом, на длине прибора можно выделить участки модуляции по энергии (рефлектор), фазовой коррекции сгустка (первая половина рабочей части $3 \mathrm{C}$ ) и энергоотбора (участок ЗС вблизи места высадки пучка). При этом группировка пучка развивается на всем протяжении от рефлектора до места высадки. Поскольку передача энергии от электронного пучка происходит на коротком финальном участке и в основном в попутную волну, такой прибор можно рассматривать как релятивистский твистрон [11].

Моделирование показало, что прибор работоспособен и при пониженных ведущих магнитных полях (ниже области циклотронного поглощения встречной волны): в магнитном поле $0.65 \mathrm{~T}$ эффективность генерации составила $42 \%$.

\section{Список литературы}

[1] Коровин С.Д., Куркан И.К., Ростов В.В., Тотьменинов Е.М. // Изв. вузов. Радиофизика. 1999. Т. 42. № 12. C. 1189-1196.

[2] Коровин С.Д., Полевин С.Д., Ройтман А.М., Ростов В.В. // Письма в ЖТФ. 1992. Т. 18. Вып. 8. С. 63-67.

[3] Ковалев Н.Ф., Петрухина В.И. // Электроника СВЧ. 1977. № 7. C. 102-105.

[4] Zaigao Chen, Jianguo Wang, Yue Wang, Hailiang Qiao, Dianhui Zhang, Weijie Guo // Phys. Plasmas. 2013. Vol. 20. P. 113103.

[5] Тараканов В.П. // Математическое моделирование. Проблемы и результаты. М.: Наука, 2003. С. 456- 476.

[6] Кицанов С.А., Климов А.И., Коровин С.Д., Куркан И.К., Пегель И.В., Полевин С.Д. // ЖТФ. 2002. Т. 72. Вып. 5. C. $82-90$.

[7] Totmeninov E.M., Klimov A.I, Kurkan I.K., Polevin S.D., Rostov V.V. // IEEE Trans. Plasma Sci. 2008. Vol. 36. N 5. P. 2609-2612.

[8] Totmeninov E.M., Kitsanov S.A., Vykhodtsev P.V. // IEEE Trans. Plasma Sci. 2011. Vol. 39. N 4. Part 2. P. 1150-1153.

[9] Пегель И.В. // Изв. вузов. Физика. 1996. № 12. С. 62-83.

[10] Denisov G.G., Lukovnikov D.A., Samsonov S.V. // Int. J. Infrared Mill. 1995. Vol. 16. N 4. P. 745-752.

[11] Ковалев Н.Ф., Кольчугин Б.Д., Кротова 3.Н. // Радиотехника и электроника. 1975. Т. 20. № 12. С. 2636-2637. 